\title{
CLOSING THE HUMAN-MACHINE DISCONNECT: DESIGN REQUIREMENTS FOR TWO EXTREME COMPANIONS FOR FUTURE AUTONOMOUS MOBILITY
}

\author{
P. Kong ${ }^{1, \bigotimes}, \mathrm{H}$. Cornet $^{1}$ and F. Frenkler ${ }^{2}$ \\ ${ }^{1}$ TUMCREATE Ltd, Singapore, ${ }^{2}$ Technical University of Munich, Germany \\ $\triangle$ penny.kong@tum-create.edu.sg
}

\begin{abstract}
In an era of increased automation in commuters' everyday life, addressing the disconnect caused by taking the human out of the loop is critical. This paper describes the development of two extreme Companions for a public autonomous bus interface in Singapore. Attributes were identified in a benchmarking study and tested in a survey to investigate local users' key preferences for must-have and ideal-to-have traits and Companion qualities. Finally, two contrasting Companion concepts are proposed and design strategies considering user expectations of Companions versus humans are discussed.
\end{abstract}

Keywords: human-centred design, industrial design, empirical studies, human-machine interfaces, human-robot interaction

\section{Introduction}

Increasing automation in the world today is moving towards a society where humans are taken out of the loop in ubiquitous scenes from daily life, such as public transportation. The future of mobility at present steers towards the automation of vehicles, whether in private or shared mobility applications. Bissell et al. (2020) argue that research into vehicle automation focuses too heavily on engineering challenges or a narrow range of social science issues, neglecting the social impacts and meanings of AVs in our daily lives. Indeed, some studies showed that the lack of a human driver or loss of control causes anxiety or distrust (Howard and Dai, 2014; Schoettle and Sivak, 2014). Feelings of anxiety discourage women, in particular, from using automated cars (Hohenberger et al., 2016). Similarly, while autonomous vehicles (AVs) increase access to mobility, older adults may instead experience an adverse effect with regards to the provision of physical assistance or the lack of a human touch (Ulahannan et al., 2019). Passengers today rely on the bus driver to not only operate the vehicle, but to provide information on routes and act as a first line of contact between the service operator and the passenger in extraordinary situations. In an autonomous bus system, the absence of the human driver leads to a gap in the customer care and information provision dimensions.

Thus, we propose to design an interface connecting the autonomous bus and passenger, termed a Virtual or Robot Companion for future autonomous mobility, which aims to evoke positive emotions in people. Given the wide range of concepts available for human-machine interfaces (HMI), it was difficult to ascertain what should or should not be included in the design brief for the Companion, which has the specific use case of $\mathrm{AV}$-passenger communication on public transport. Desmet et al. 
(2001, p. 35) state "... to understand how products elicit emotions, it must be known what specific concerns people have regarding products." This paper describes the methods used to identify local users' key preferences for a Virtual or Robot Companion for public autonomous transport in Singapore. As an exploratory study for design development, we used the Kano model (Kano et al., 1984) to distinguish the importance of various functions so that design tasks could be prioritised. The form of the Companion will be based on the functional requirements identified in this study and could thus take shape as a digital interface or physically-embodied robot. Regardless, it should be integrated within the infrastructure of an AV system for public transport where it can be accessed by any and all passengers.

\section{Literature review}

\subsection{Autonomous vehicle human-machine interfaces}

Existing work on human-AV interaction trends towards vehicle to driver communication (Damiani et al., 2009; Debernard et al., 2016; Häuslschmid et al., 2017) with advanced driver assistance systems (Akamatsu et al., 2013) or to build relationships between the driver and the vehicle (Etherington, 2017). Personalised virtual companions currently being researched in several EU-funded projects focus on the driver-vehicle interface, which aim to nudge private vehicle drivers towards safer, calmer driving behaviour. Other research focuses on vehicle to pedestrian (Mahadevan et al., 2018; Rasouli and Tsotsos, 2019; Stadler et al., 2019) or vehicle to vehicle communication (Bengler et al., 2014). In the area of vehicle to passenger communication, a case study on a public autonomous bus described HMI concepts for vehicle navigation and route information (Stadler et al., 2019). Vehicle to passenger solutions tend to focus on providing vehicle navigation information more appropriate for private vehicle ownership models. Because there is a dearth of research in vehicle-to-passenger communication concepts for AVs used in public transport, further investigation is warranted to define design requirements.

\subsection{The Kano model of product innovation}

The Kano model (Kano et al., 1984) defines three levels of product quality to achieve greater customer satisfaction and was proposed as a tool for planning new products. The three basic levels are defined as follows (Horton and Goers, 2019; Sauerwein et al., 1996):

- Must-be requirements: basic qualities which cause dissatisfaction in the user when absent. On the other hand, the presence of such qualities does not induce greater satisfaction.

- Satisfy requirements: qualities which users focus on to evaluate and compare competing products and are directly related to user satisfaction and dissatisfaction.

- Delight requirements: qualities which have the greatest influence on user satisfaction. The absence of such qualities does not cause user dissatisfaction.

Application of the Kano model to user research usually involves asking a pair of questions per attribute. The first question collects the user's reaction to the presence of the attribute (functional form of the question), while the second asks about their reaction to the absence of the attribute (dysfunctional form). The Kano model, in combination with Hofstede's Cultural Model, was used to identify cultural preferences for automotive HMI design features (Khan et al., 2016). The study compared the preferences of users from India and the United Kingdom. Findings suggested that cultural differences influence user preferences for features - some features which UK drivers were Indifferent to were considered Must-be or Attractive by Indian drivers. Since the Companion serves in a position interacting with multiple user types, it is important to discover the key preferences of users in Singapore.

\section{Methodology}

There were three main parts to the study beginning with a benchmarking exercise, followed by a survey and design concept development. Figure 1 depicts a summary of the methodology. 


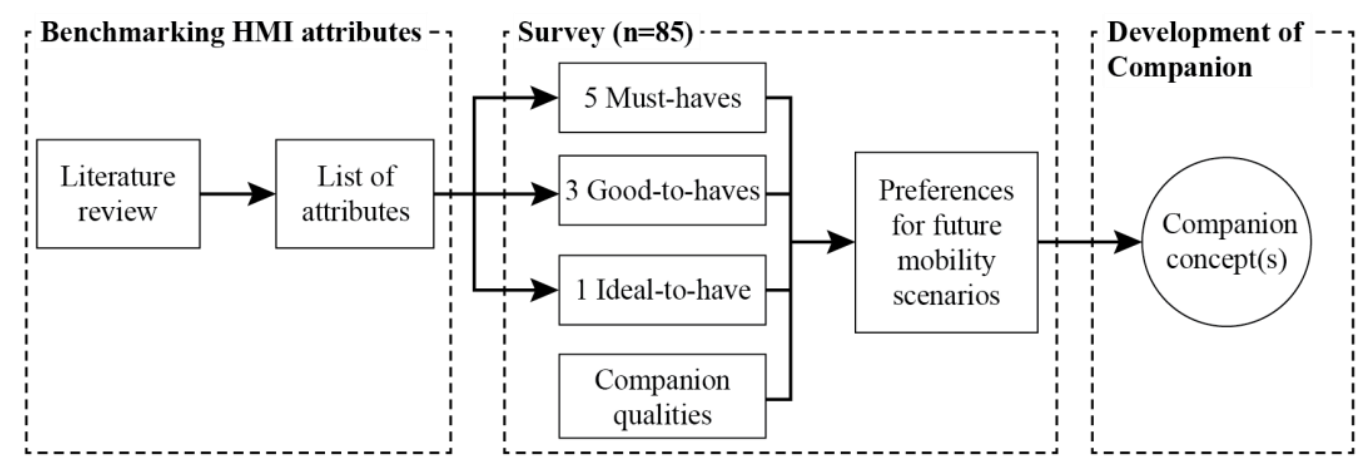

Figure 1. A summary of the study methodology

\subsection{Benchmarking $\mathrm{HMl}$ attributes}

A benchmarking study was initiated to collect a list of attributes of HMIs, ranging from smartphone apps to robots, through a literature review consisting of scholarly sources, grey literature and commercial products and concepts. The scope of HMI concepts was not restricted to allow for an open-ended exploration of potential functions and design characteristics. Concepts were broadly sorted based on their form factors, then described through their purpose, functions and other characteristics. Common attributes within each form factor were consolidated. The individual lists from each form factor were then combined, with duplicates merged, to consolidate a final list of attributes. Observations of services (i.e. functions) performed by human staff such as a bus driver were added to the list of possible functions for a Virtual or Robot Companion. The attributes were analysed for sorting into functional categories (van Boeijen et al., 2013), which defined the role they would play in a future Companion. With the final list of attributes, a survey questionnaire was designed.

\subsection{Survey design}

A final list of attributes was selected for the survey based on their relevance to the context, i.e. autonomous mobility and public transport. These attributes were concerned with the practical functions of the Companion, which are physical aspects of the artefact at the user level (Heufler, 2004). These were presented as part of a section for subjects to choose attributes of their desired Virtual or Robot Companion. Based on the three levels of product attributes in the Kano model, subjects were asked to choose five must-haves (corresponding to Must-be), three good-to-haves (Satisfy) and one ideal-to-have (Delight) from the attributes list. The number of attributes were decided based on two self-prescribed guidelines. Firstly, to have an ascending number of features from one ideal-to-have to more core features, i.e. must-haves. Secondly, the total number of attributes chosen by respondents should not exceed half of the total number of attributes available for selection. This method was used instead of the pairwise format suggested by the Kano model, where each item is measured in terms of function and dysfunction (Sauerwein et al., 1996), to reduce the survey length. Results from the survey focused on the must-haves and ideal-to-haves for the development of the two extreme Companion concepts as discussed below. In order to develop the Companion's qualities, questions were asked about the personality (sociable or informative), appearance of the Companion (more human-like, animal-like or abstract), form factor (screen display, mobile app, voice assistant, hologram, physical robot), and input and output mode (e.g. via voice commands, text inputs and outputs). Subjects were then presented with four autonomous public bus scenarios and tasked to choose between receiving assistance by the Virtual/Robot Companion they had designed in the previous section, or a human service staff via phone or video call. The four scenarios were:

1. Wrong Bus: You realise you are on the wrong bus and need to find out how to get to your destination.

2. Unwell: You feel light-headed and you need help.

3. Need Information: You need information on where to alight from the autonomous bus.

4. Multiple Buses: You are at a bus stop and multiple autonomous buses arrive. You are not sure which bus to board. 
By presenting the scenarios only after participants had already selected attributes and qualities for their ideal Companion, we could avoid priming participants and use the results for desired attributes and qualities to study people's organic perceptions of the roles of future Companions. Additional questions collected demographic information such as age, gender and education and general opinions on autonomous vehicles. The survey took approximately 15-20 minutes to complete.

\subsection{Development of two extreme companion concepts}

Based on the survey results, the top-ranking attributes for the must-have and ideal-to-have categories were used to develop two extreme concepts for a Virtual or Robot Companion. The middle tier of attributes was left out to delineate clearly antithetical combinations. This makes explicit and obvious their differences when presented to subjects for evaluation in order to isolate attributes and their relationship to emotional responses and user satisfaction. In developing the two extreme concepts, the higher-ranking attributes in each category were used as characteristics. Each Companion had traits from either the must-have or ideal-to-have list. The two concepts were then evaluated internally to ensure that there were no contradictions in each set. The attributes guided the choice of form factor.

\section{Results}

\subsection{Consolidated list of attributes from benchmarking}

After gathering the different HMI concepts, the concepts were sorted into categories based on form factors. The form factors were mobile applications (e.g. Whim (MaaS Global Oy, 2019)), digital assistants (e.g. Yui AI (Toyota, 2018)), holographic assistants (e.g. Vntana (Vntana, 2017)) and robots (e.g. Nadine Social Robot (Institute for Media Innovation (IMI), 2019)). The status quo, i.e. with human service staff, was also considered. A total of nineteen attributes were selected based on their relevance to mobility interfaces and consolidated into Table 1.

Table 1. The consolidated list of attributes compiled from the benchmarking investigation

\begin{tabular}{ll}
\hline Form Factors & Attributes \\
\hline Status Quo: Human & Physical assistance \\
Staff & Answer travel-related queries \\
& Take complaints or feedback \\
\hline Multimodal & Suggest nearby places of interest \\
Mobility Mobile & News, entertainment or weather updates \\
Apps & Real-time updates on traffic conditions \\
& Real-time updates on departure times \\
& Share location with others \\
\hline Digital Assistants & Emotion recognition \\
& Ability to make custom requests \\
& Artificial intelligence (AI) for travel personalisation \\
& Route prediction based on trip history \\
& Facial recognition \\
\hline Holograms & Capture real-time data on users \\
\hline Robots & Modular and configurable design \\
& Fitted with a personality \\
& Fitted with facial expressions \\
& Provide notifications and alerts \\
& Fast, reliable and durable \\
\hline
\end{tabular}

Results from the benchmarking show a range of attributes generally centred around the provision of information, customer care or personalisation. Following the benchmarking, we hypothesised that users would consider functional attributes to be core features (i.e. Must-be), while other attributes would be considered as good-to-haves (i.e. Satisfy) or ideal-to-haves (i.e. Delight). 


\subsection{Attribute ranking}

Ninety-five participants completed the survey, which was conducted through convenience sampling at a university engineering fair. Most respondents were either students (61\%) or employed full-time $(32 \%)$. For the item on attribute ranking, responses were discarded where respondents did not select the exact number of items in each category. Thus, forty-five responses $(42 \%$ female; mean age $=27.6$ years, $\mathrm{SD}=8.9$ years) were accepted for analysis. Some items appeared in more than one category. For example, 'ability to make custom requests' appeared in all three categories of must-have, good-tohave and ideal-to-have. Figure 2 and Figure 3 show the ten most preferred attributes in the must-have and ideal-to-have categories.

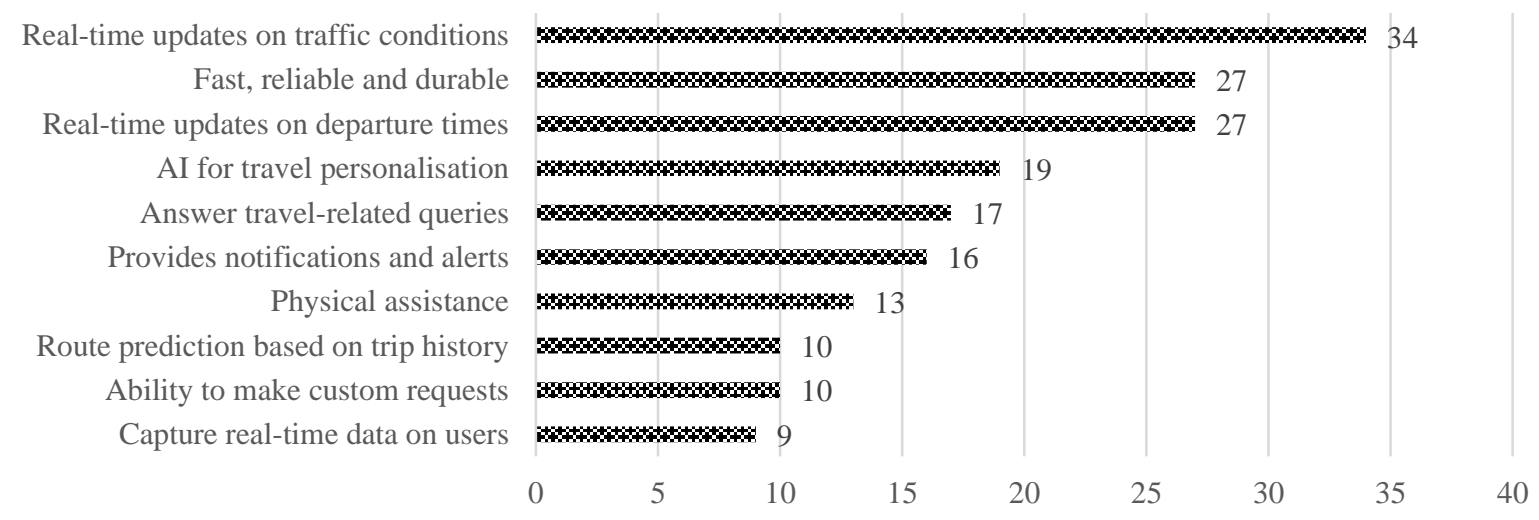

Figure 2. The ten most desired must-haves for a Companion $(n=45)$

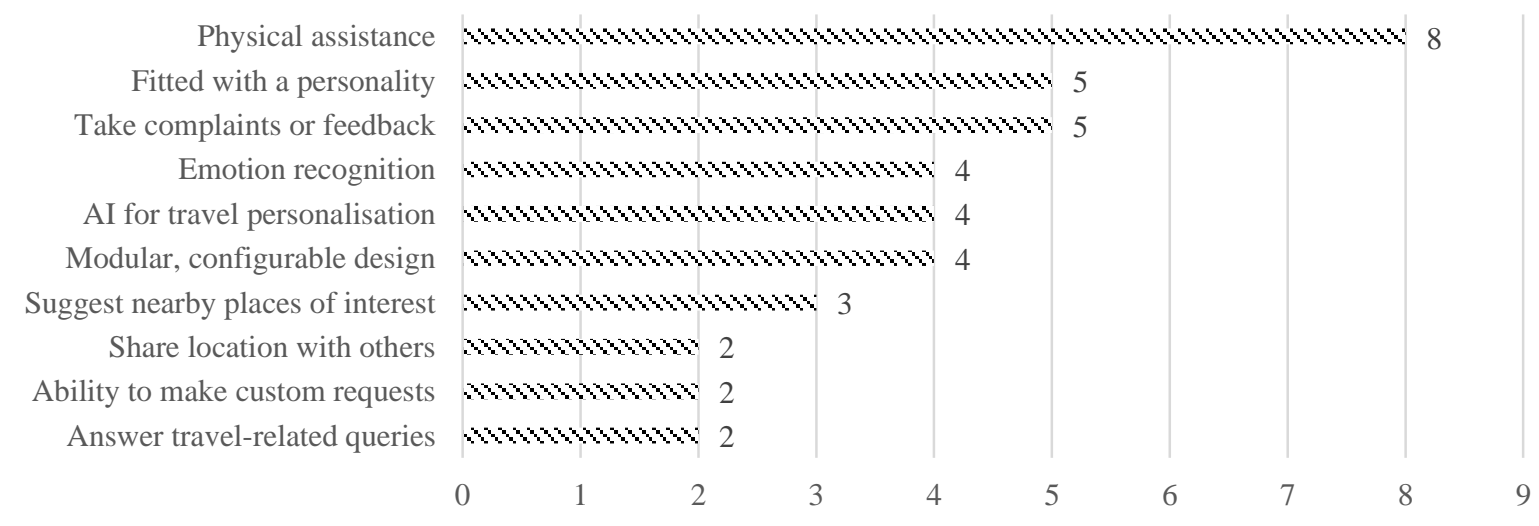

Figure 3. The ten most desired ideal-to-haves for a Companion $(n=45)$

Functional attributes which aid the traveller in their mobility journey were popular must-haves among respondents. Most of these attributes are available today as part of the public transport system in Singapore, so the results serve to reinforce the importance of these features as a basic level of service. On the other hand, the presence of personalisation and prediction features - such as 'artificial intelligence (AI) for travel personalisation' and 'provides notifications and alerts' - suggest a preference for more innovative tools to make public transport journeys more tailored to the individual and hassle-free. It could be due to the prevalence of smartphone use and mobile navigation apps such as Google Maps. Such willingness to share personal trip data could be an important signifier of acceptance and trust in a future Companion service.

Results for the ideal-to-haves show a tendency towards attributes that are non-essential, but which enhance the travel experience. Companions attributes which are expressive, personal and aid communication - either with the service operator or friends - feature prominently in this set. Physical assistance is the most highly-ranked. Today, bus drivers or MRT station ushers provide this service, which is something mobile apps cannot reproduce. Since this item and two others (i.e. AI and custom requests) also appeared on the must-haves list, these are strongly recommended for inclusion in the list of design requirements. 


\subsection{Preferences for assistance in future mobility scenarios}

Eighty-five responses $(\mathrm{n}=85 ; 43 \%$ female; mean age $=27.9$ years, $\mathrm{SD}=8.7$ years) were used to analyse the following survey items; ten entries were deemed invalid as they had incorrectly selected more than one response per scenario. For the autonomous bus scenarios, participants picked the Virtual or Robot Companion that they had designed as their preferred choice of assistance instead of human service staff in three out of four scenarios (Figure 4). Participants showed a strong preference for the Companion - over a human service staff - to provide assistance, except for the Unwell scenario. When prompted to explain why they did not pick the Companion they had designed earlier for any of the scenarios, one participant said, "When [it] comes to immediate health concerns, I prefer human-based interaction." The uncertainty of the capabilities of AI influenced their answers. Other responses echoed the sentiment, preferring a human being who can react quickly and appropriately no matter the emergency. Such statements hint at a threshold where user trust or acceptance falls. In this case, the urgency and complexity of the situation and the need for immediate medical attention influenced participants' responses.

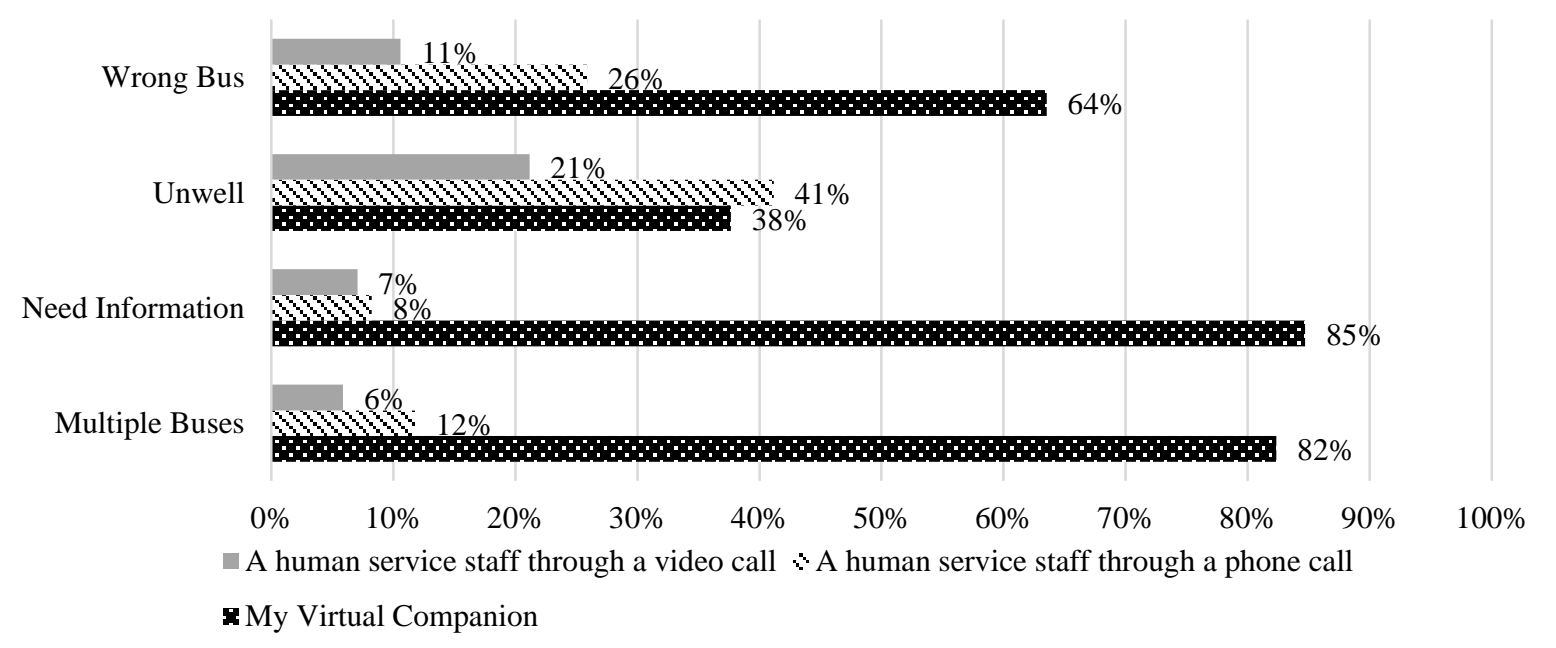

Figure 4. Choice of assistance in four autonomous bus scenarios $(n=85)$

\subsection{Companion qualities and role expectations}

Ten entries were deemed invalid as they had incorrectly selected more than one response per scenario, leaving eighty-five respondents for analysis. When it comes to form factors, the hologram was the most popular choice (35\%), followed by a screen display (24\%). Robots and voice assistants were tied (15\% each). A majority of respondents $(65 \%)$ preferred a more human-like appearance over a more abstract one (26\%). In terms of input and output to communicate with the Companion, participants could choose multiple options. Results showed that respondents preferred voice and touchscreen inputs, and voice and graphics outputs. The results suggest physical button inputs or text inputs via keyboards should be avoided. Considering the mobility context, convenience and ease of use could be two factors influencing participants' choices. Furthermore, participants preferred a more informative (66\%) Companion over a sociable Companion. However, when comparing participants who had chosen only the Companion for all scenarios to those who had chosen human assistance for at least one scenario, some differences emerged. Figure 5 shows the distribution of preferences for an informative versus sociable Companion based on participants' choice of assistance. The quotes in Figure 5 express a possible interpretation by designers while developing the Companion for the two groups of users (i.e. those who still need human assistance in some scenarios and those who do not).

Twenty-five respondents ( $29 \%$ of valid responses; mean age $=25.7$ years, $\mathrm{SD}=7.3$ years $)$ did not need human assistance for any of the four scenarios. Their preference for an informative or sociable Companion was split evenly (52\% more informative and $48 \%$ more sociable). By contrast, participants who needed human assistance in at least one scenario $(n=60$; mean age $=28.9$ years, $S D=9.1$ years $)$ 
had a distinct preference (72\%) for a more informative Companion. The two groups are also either slightly younger or older than the sample average (27.9 years). The trends suggest two diverging perspectives for future Companions. Some respondents preferred the attribute 'more informative' when envisioning their ideal Companion, which could be because they tended to view Companions as a tool to assist human staff and enhance the services that they, i.e. human staff, provide. In such an instance, the human staff naturally fulfils a 'sociable' role. The other group of respondents might have perceived Companions as replacing human roles completely, which is reflected in their choice of the Companion for all the scenarios. The reason for the lack of a clear trend for either a more informative or sociable trait suggests this group perceives both traits as equally important. Based on the results, we posit that preserving a social connection is still crucial even in the face of automation.

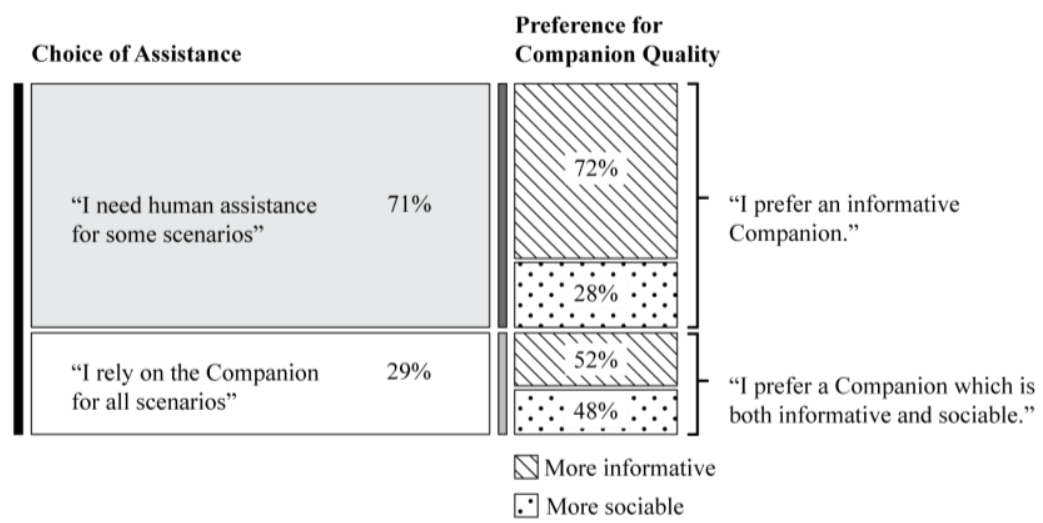

Figure 5. Choice of assistance and preferences for an informative or sociable Companion $(n=85)$

\subsection{Development of two companion personas}

Based on the design requirements from the survey results, two concepts were developed for evaluation by users (Figure 6 ). The two concepts are:

a) Based on ideal-to-have attributes: A sociable, humanoid Robot Companion which offers personalisation and can provide physical assistance but does not provide information

b) Based on must-have attributes: An impersonal, more reactive (as opposed to active) and informative Virtual Companion, which is screen-based

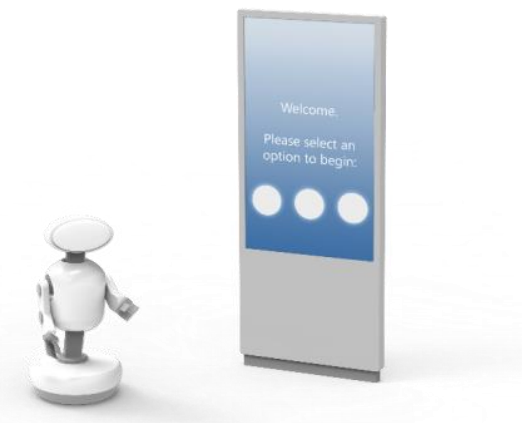

Figure 6. Early concepts for the Robot and Virtual Companions

The Virtual Companion features more on providing practical information, as opposed to the Robot Companion's social camaraderie. Communication with the Robot Companion is via voice while the Virtual Companion is activated via a touchscreen. The provision of physical assistance as the top ideal-tohave supported the use of a robot over a hologram, although hologram was the most popular form factor.

\section{Discussion}

The most highly preferred attributes in the must-haves list relate to the servicing of basic mobility functions, such as information on bus and train arrivals and traffic conditions. These reflect people's 
tendencies toward instrumental elements with practical benefits (Guell et al., 2012). With $53.3 \%$ of local residents relying on public transport as their usual mode of transport (Singapore Department of Statistics, 2015), the provision of core mobility services is crucial. The presence of must-have items which already exist today could also be due to a tendency for people to relate to the familiar. However, to avoid falling into the 'empathy trap' (Mattelmäki et al., 2014), these preferences should not be accepted at face value, which risks stagnancy and lack of product or service innovation. Designers should instead understand the rationale behind these preferences to translate these user needs and wants into serviceable features for future products or services in future contexts. Additionally, the presence of items relating to personalisation and AI suggests that the must-haves list of users will evolve over time, as more of such innovative ideas are introduced into the mainstream and become entrenched as a minimum level of product or service offerings. Results echo the life cycle of attributes (Löfgren et al., 2011), which stated that Satisfy and Delight requirements could gradually evolve to become Must-be attributes. Generally, the results show that must-haves lean towards traffic or route-related information provision while ideal-to-haves trend towards customer care components. The latter relate to the provision of an extra, non-essential layer of information or entertainment, as well as personalisation (e.g. making custom requests, remembering preferences and routes). Many ideal-to-have items reflect the services and abilities provided by human service staff today, while several must-haves relating to information can be achieved with mobile apps on smartphones. Respondents might, again, be unconsciously evaluating the nineteen attributes based on services they are accustomed to.

The choice of assistance for the four scenarios could be an indicator of the trust or acceptance participants have in future Companions for autonomous mobility. Two-thirds of respondents preferred some level of human assistance, suggesting that while some level of trust is shown in the Virtual or Robot Companion, ultimately, human staff are perceived as more reliable in scenarios such as medical situations. It raises the question of how a Virtual or Robot Companion for future autonomous mobility can be designed to provide benefits beyond the abilities of human staff or smartphones in order to justify its development. Existing studies point to some design strategies to overcome lack of trust. For instance, anthropomorphic characteristics in AVs have been found to increase trust (Waytz et al., 2014) and are a preferred trait in pedestrian HMIs during the transition to AVs (Verma et al., 2019). Automated systems which appear to share the same goals as their human users may also be perceived as more trustworthy (Verberne et al., 2012). Another design strategy considering participants' readiness in taking humans out of the loop could lie in defining the roles of Companions in the transition to an automated society. The distinction between respondents' preferences for more informative or sociable traits suggests two paths for design. When designing a Companion as a support for human staff, more practical attributes could be important, while Companions working alone might need to offer both practical and social functions. Interestingly, a study by Nordhoff et al. (2017) found that in AVs, supervision from an external control room was preferred to supervision by an on-board steward (both human). A possible explanation could be the small, confined space of the 8person autonomous shuttle. The size of the vehicle - whether it be an AV, present-day public bus or MRT train - relative to the user perception of personal space could be an influencing factor. The role of the Companion could thus fulfil this gap balancing privacy and the provision of customer care. With the development of the two Companions, further work will centre around their evaluation in an application of design fiction as proposed by Lindley and Coulton (2015) to investigate people's preferences in context.

Several limitations could be addressed to improve the methodology and results for generalisability. The provision of a list of attributes - as opposed to an open-ended question seeking ideas - might have restricted the possibilities of Companion features and characteristics. Due to the long list of nineteen attributes presented in the survey, participants were confused about how to answer the question. As a result, many responses were discarded and a small sample of valid responses remained, reducing the power of the results. Further work can be conducted with a refined survey questionnaire to clarify question instructions. The use of convenience sampling also limits the results as the age group featured a disproportionate number of younger respondents. The slight difference in ages when choosing Companion versus human assistance for the four scenarios suggest a direction in this line of questioning. 


\section{Conclusion}

The study has successfully identified key design requirements for a Virtual and Robot Companion for future autonomous mobility for users in Singapore. By way of a benchmarking exercise and survey based on the Kano model, product features were identified on two levels: a core set of attributes (i.e. must-haves) and a value-added set (i.e. ideal-to-haves). Results from the ranking of the benchmarked attributes and qualities led to two strategic design directions - and consequently, set of attributes - for future Companions depending on their role. For roles which complement human staff, a Virtual Companion focusing on practical support, such as information provision, suffices. Where humans are completely replaced, a Robot Companion which can foster social connection is preferable. As a next step, the two Companions will be tested with users in a study based on emotional design, with the goal of defining a link between the two layers of attributes and user emotion and satisfaction.

\section{Acknowledgements}

The authors thank Jerome Goh for his assistance in data collection and Niklas Forchhammer for the 3D renders. This study is part of a research programme, TUMCREATE Phase 2, funded by the National Research Foundation (NRF) Singapore under its Campus for Research Excellence and Technological Enterprise (CREATE) programme.

\section{References}

Akamatsu, M., Green, P. and Bengler, K. (2013), "Automotive Technology and Human Factors Research: Past, Present, and Future", International Journal of Vehicular Technology, Vol. 2013, pp. 1-27. https://doi.org/ $10.1155 / 2013 / 526180$

Bengler, K. et al. (2014), "Three Decades of Driver Assistance Systems: Review and Future Perspectives”, IEEE Intelligent Transportation Systems Magazine, Vol. 6 No. 4, pp. 6-22. https://doi.org/10.1109/MITS.2014. 2336271

Bissell, D. et al. (2020), “Autonomous automobilities: The social impacts of driverless vehicles", Current Sociology, Vol. 68 No. 1, pp. 116-134. https://doi.org/10.1177/0011392118816743

Damiani, S., Deregibus, E. and Andreone, L. (2009), "Driver-vehicle interfaces and interaction: where are they going?", European Transport Research Review, Vol. 1 No. 2, pp. 87-96. https://doi.org/10.1007/s12544009-0009-2

Debernard, S. et al. (2016), "Designing Human-Machine Interface for Autonomous Vehicles", IFACPapersOnLine, Vol. 49 No. 19, pp. 609-614. https://doi.org/10.1016/j.ifacol.2016.10.629

Desmet, P., Overbeeke, K. and Tax, S. (2001), "Designing Products with Added Emotional Value: Development and Appllcation of an Approach for Research Through Design”, The Design Journal, Vol. 4 No. 1, pp. 3247. https://doi.org/10.2752/146069201789378496

Etherington, D. (2017), “Here's what it's like to drive with Toyota's Yui AI in-car assistant”, TechCrunch, available at: https://techcrunch.com/2017/01/06/heres-what-its-like-to-drive-with-toyotas-yui-ai-in-car-assistant/ (acce ssed 19 April 2018).

Guell, C. et al. (2012), "Towards a differentiated understanding of active travel behaviour: Using social theory to explore everyday commuting", Social Science and Medicine, Vol. 75 No. 1, pp. 233-239. https://doi.org/10.1016/j.socscimed.2012.01.038

Häuslschmid, R. et al. (2017), "Supporting Trust in Autonomous Driving", Proceedings of the 22nd International Conference on Intelligent User Interfaces - IUI '17, pp. 319-329. https://doi.org/10.1145/ 3025171.3025198

Heufler, G. (2004), Design Basics, translated by Zettinig, S.M., Niggli Verlag AG, Sulgen, Zurich.

Hohenberger, C., Spörrle, M. and Welpe, I.M. (2016), "How and why do men and women differ in their willingness to use automated cars? The influence of emotions across different age groups", Transportation Research Part A: Policy and Practice, Vol. 94, pp. 374-385. https://doi.org/10.1016/j.tra.2016.09.022

Horton G. and Goers, J. (2019), A Revised Kano Model and its Application in Product Feature Discovery, University of Magdeburg. Magdeburg, Germany.

Howard, D. and Dai, D. (2014), "Public Perceptions of Self-driving Cars: The Case of Berkeley, California", 93rd Annual Meeting of the Transportation Research Board, Vol. 2014, p. 21.

Institute for Media Innovation (IMI). (2019), "Nadine Social Robot", available at: https://imi.ntu.edu.sg/IMIResearch/ ResearchAreas/Pages/NadineSocialRobot.aspx (accessed 6 November 2019).

Kano, N. et al. (1984), “Attractive Quality and Must-Be Quality”, Journal of The Japanese Society for Quality Control, Vol. 14 No. 2, pp. 147-156. 
Khan, T., Pitts, M. and Williams, M.A. (2016), "Cross-Cultural Differences in Automotive HMI Design: A Comparative Study Between UK and Indian Users' Design Preferences”, Journal of Usability Studies, Vol. 11 No. 2, pp. 45-65.

Lindley, J. and Coulton, P. (2015), "Back to the future: 10 years of design fiction", ACM International Conference Proceeding Series, pp. 210-211. https://doi.org/10.1145/2783446.2783592

Löfgren, M., Witell, L. and Gustafsson, A. (2011), "Theory of attractive quality and life cycles of quality attributes", TQM Journal, Vol. 23 No. 2, pp. 235-246. https://doi.org/10.1108/17542731111110267

MaaS Global Oy (2019), “Whim”, available at: https://whimapp.com/ (accessed 6 November 2019).

Mahadevan, K., Somanath, S. and Sharlin, E. (2018), "Communicating awareness and intent in autonomous vehicle-pedestrian interaction", CHI '18 Proceedings of the 2018 CHI Conference on Human Factors in Computing Systems, ACM, Montreal, QC, Canada, available at: https://doi.org/10.1145/3173574.3174003

Mattelmäki, T., Vaajakallio, K. and Koskinen, I. (2014), “What Happened to Empathic Design?”, Design Issues, Vol. 30 No. 1, pp.67-77. https://doi.org/10.1162/desi_a_00249

Nordhoff, S. et al. (2017), "User acceptance of automated shuttles in Berlin-Schöneberg: A questionnaire study", Transportation Research Part F: Traffic Psychology and Behaviour, Vol. 58 No. August, pp. 843-854. https://doi.org/10.1016/j.trf.2018.06.024

Rasouli, A. and Tsotsos, J.K. (2019), "Autonomous Vehicles That Interact With Pedestrians: A Survey of Theory and Practice", IEEE Transactions on Intelligent Transportation Systems, IEEE, pp. 1-19. https://doi.org/10.1109/tits.2019.2901817

Sauerwein, E. et al. (1996), "The Kano Model: How to Delight Your Customers", International Working Seminar on Production Economics, Vol. 1 No. 4, pp. 313-327.

Schoettle, B. and Sivak, M. (2014), "A survey of public opinion about connected vehicles in the U.S., the U.K., and Australia", 2014 International Conference on Connected Vehicles and Expo, ICCVE 2014 Proceedings, pp. 687-692. https://doi.org/10.1109/iccve.2014.7297637

Singapore Department of Statistics. (2015), Highlights of General Household Survey 2015, Singapore.

Stadler, S. et al. (2019), "Designing Tomorrow' s Human-Machine Interfaces in Autonomous Vehicles: An Exploratory Study in Virtual Reality", Proceedings of the 5th International Augmented Reality and Virtual Reality Conference.

Stadler, S. et al. (2019), "A Tool, not a Toy: Using Virtual Reality to Evaluate the Communication Between Autonomous Vehicles and Pedestrians", in tom Dieck, M.C. and Jung, T.H. (Eds.), Augmented Reality and Virtual Reality, Springer Nature Switzerland AG, Manchester, England, pp. 203-216. https://doi.org/ 10.1007/978-3-030-06246-0_15

Toyota. (2018), "Toyota Concept-i | The Car of the Future", available at: https://www.toyota.com/concept-i/ (accessed 19 April 2018).

Ulahannan, A. et al. (2019), "Using the Ideas Café to Explore Trust in Autonomous Vehicles", in Ho, A.G. (Ed.), Proceedings of the AHFE 2018 International Conference on Human Factors in Communication of Design, Vol. 796, Springer International Publishing, Orlando, Florida, USA, pp. 3-14. https://doi.org/10. 1007/978-3-319-93888-2

van Boeijen, A. et al. (Eds.). (2013), Delft Design Guide: Design Methods, BIS Publishers, Amsterdam.

Verberne, F.M.F., Ham, J. and Midden, C.J.H. (2012), “Trust in Smart Systems”, Human Factors, Vol. 54 No. 5, pp. 799-810. https://doi.org/10.1177/0018720812443825

Verma, H. et al. (2019), "Engaging Pedestrians in Designing Interactions with Autonomous Vehicles", CHI EA '19 Extended Abstracts of the 2019 CHI Conference on Human Factors in Computing Systems, ACM, Glasgow, Scotland UK, available at: https://doi.org/10.1145/3290607.3312864

Vntana. (2017), "Vntana", available at: https://vntana.com/ (accessed 6 November 2019).

Waytz, A., Heafner, J. and Epley, N. (2014), "The mind in the machine: Anthropomorphism increases trust in an autonomous vehicle", Journal of Experimental Social Psychology, Elsevier Inc, Vol. 52, pp. 113-117. https://doi.org/10.1016/j.jesp.2014.01.005 